\title{
TOPOLOGICAL ENTROPY OF FOLIATION PRESERVING DIFFEOMORPHISMS
}

\author{
KOICHI YANO
}

\begin{abstract}
We give some estimations for the topological entropy of diffeomorphisms preserving foliated structures, one of which partially supports the entropy conjecture.
\end{abstract}

Introduction. Throughout this note, $M$ denotes a closed $m$-dimensional $C^{\infty}$ manifold, $\mp$ an orientable $p$-dimensional $C^{\infty}$-foliation of $M$ and $f$ a diffeomorphism of $M$ which preserves $₹$. Let $h(f)$ denote the topological entropy of $f$ and $\rho(f)$ the volume expanding ratio of $f$, which will be defined in $\S 1$. Now our result is stated as follows:

THEOREM $1 . h(f) \geq \rho(f)$.

To state Theorem 2, we need the notion of foliation cycles, which was first introduced by Plante [2] as holonomy invariant measures and reformulated by Sullivan [4] as foliation cycles. Let $F$ denote the linear subspace of $H_{p}(M ; \mathbf{R})$ consisting of homology classes represented by foliation cycles and $\operatorname{sp}\left(\left.f_{*}\right|_{F}\right)$ the spectral radius of the induced linear mapping $\left.f_{*}\right|_{F}: F \rightarrow F$. Then, as a corollary to the theorem above, we get the following result which partially supports the "entropy conjecture". (See [3] for the entropy conjecture.)

THEOREM 2. $h(f) \geq \log \operatorname{sp}\left(\left.f_{*}\right|_{F}\right)$.

The author would like to thank N. Tsuchiya, T. Inaba and K. Sasano for helpful conversations.

1. Definitions and notation. Fix a Riemannian metric on $M$. Let $d$ and $d_{L}$

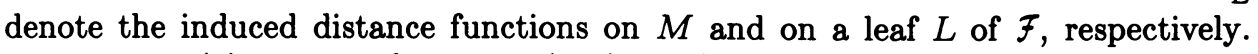
Hereafter $B_{r}(x)$ denotes $\{y \in M ; d(x, y) \leq r\}$ and similarly $D_{r}(x)$ denotes $\{y \in$ $\left.L ; d_{L}(x, y) \leq r\right\}$ and is called an $r$-leaf-ball, where $L$ is the leaf which contains $x$. Also fix a family of distinguished charts $\left\{D_{i}^{p} \times D_{i}^{m-p}\right\}_{i=1, \ldots, N}$ whose interiors cover $M$ and an associated partition of unity $\left\{\lambda_{i}\right\}$. We put $P_{z}=D_{i}^{p} \times\{z\}$ and call this set a plaque. If necessary, taking a refinement, we may assume that for every plaque $P_{z}$ and every index $j$, the set $P_{z} \cap D_{j}^{p} \times D_{j}^{m-p}$ is contained in some plaque $P_{z^{\prime}}$ for $z^{\prime} \in D_{j}^{m-p}$.

First, recall the definition of the topological entropy according to Bowen [1]. We say that a subset $X$ of $M(n, \delta)$-spans $M$ with respect to $f$, if for any $x \in M$ there exists an $x^{\prime} \in X$ such that $d\left(f^{i}(x), f^{i}\left(x^{\prime}\right)\right)<\delta$ for $i=0, \ldots, n-1$. We put $s_{f}(n, \delta)$ to be the minimum of the cardinalities of such subsets. Then the topological entropy

Received by the editors March 13, 1980 and, in revised form, September 3, 1981.

1980 Mathematics Subject Classification. Primary 58F99.

Key words and phrases. Topological entropy, foliation. 
of $f$ is defined by the following:

$$
h(f)=\sup _{\delta>0} \limsup _{n} \frac{1}{n} \log \left(s_{f}(n, \delta)\right) .
$$

Next, we define the volume expanding ratio of $f$, denoted by $\rho(f)$.

DEFINITION.

$$
\rho(f)=\underset{n}{\limsup } \frac{1}{n} \log \left(\sup _{x \in M} \operatorname{volume}\left(f^{n}\left(D_{r}(x)\right)\right)\right) .
$$

ClAIM. The above definition is independent of the choice of $r$ 's and Riemannian metrics. Moreover, we have the following formula:

$$
\rho(f)=\underset{n}{\lim \sup } \frac{1}{n} \log \left(\sup _{z} \operatorname{volume}\left(f^{n}\left(P_{z}\right)\right)\right) .
$$

Proof. Let $F\left(P_{z}\right)=\left\{P_{z^{\prime}} ; P_{z} \cap P_{z^{\prime}} \neq \emptyset\right\}$ and define $F^{k}\left(P_{z}\right), k=0,1, \ldots$, inductively as $F^{0}\left(P_{z}\right)=\left\{P_{z}\right\}$ and $F^{k}\left(P_{z}\right)=\bigcup_{P_{z^{\prime}} \in F^{k-1}\left(P_{z}\right)} F\left(P_{z^{\prime}}\right)$. It is easy to see that the cardinality of $F^{k}\left(P_{z}\right)$ is not greater than $N^{k}$ for every plaque $P_{z}$. Let $r_{0}$ be small enough that every $r_{0}$-leaf-ball is contained in some plaque. Then, by induction on $k$, we have that if $D_{r_{0}}(x) \subset P_{z}, D_{k r_{0}}(x) \subset \bigcup_{P_{z^{\prime}} \in F^{k-1}\left(P_{z}\right)} P_{z^{\prime}}$. Thus, for any $r>0$, there is a positive integer $k$ such that

$$
\sup _{x \in M} \operatorname{volume}\left(f^{n}\left(D_{r}(x)\right)\right) \leq N^{k-1} \sup _{z} \operatorname{volume}\left(f^{n}\left(P_{z}\right)\right) \text {. }
$$

On the other hand, there exists a positive integer $K$ such that every plaque is covered by at most $K r$-leaf-balls. Then we get

$$
\sup _{\boldsymbol{z}} \operatorname{volume}\left(f^{n}\left(P_{z}\right)\right) \leq K \sup _{x \in M} \operatorname{volume}\left(f^{n}\left(D_{r}(x)\right)\right) \text {. }
$$

Since $r$ is an arbitrary positive number, we have proved the required formula and the independence of the definition from the choice of $r$ 's.

Next, let volume' $(A)$ denote the volume of a compact subset $A$ contained in some leaf of $\mathcal{F}$ with respect to another Riemannian metric on $M$. Then, by the compactness of $M$, there are positive constants $C_{1}$ and $C_{2}$ satisfying $C_{1} \operatorname{volume}(A) \leq$ volume' $(A) \leq C_{2}$ volume $(A)$ for every such $A$. Thus, for every $P_{z}$, we have

$$
C_{1} \text { volume }\left(f^{n}\left(P_{z}\right)\right) \leq \operatorname{volume}\left(f^{n}\left(P_{z}\right)\right) \leq C_{2} \text { volume }\left(f^{n}\left(P_{z}\right)\right) \text {. }
$$

This implies the independence from the choice of Riemannian metrics.

The definition of foliation cycles is as follows: Let $D_{p}$ denote the linear space consisting of $p$-dimensional smooth forms on $M$ and $D_{p}^{\prime}$ the dual of $D_{p}$. Define the closed convex cone $C$ of $D_{p}^{\prime}$ to be one that is generated by all Dirac currents at positive $p$-vectors tangent to $\mp$ and call an element of $C$ a foliation cycle, if it is closed as a current. Here "positive" means the compatibility to a fixed orientation of $\mathcal{F}$. We also call a closed current a foliation cycle if it is represented as a difference of foliation cycles defined above. The following lemma due to Sullivan [4] asserts that every foliation cycle has an expression as a transverse invariant measure.

LEMMA 1 (THEOREM I.12 OF [4]). For every foliation cycle $\alpha$, there exists a family of signed measures $\mu_{i}$ on $D_{i}^{m-p}, i=1, \ldots, N$, such that

$$
\langle\alpha, \eta\rangle=\sum_{i} \int_{D_{i}^{m-p}}\left(\int_{P_{z}} \lambda_{i} \eta\right) d \mu_{i}(z),
$$

where $\eta$ denotes an arbitrary $p$-form on $M$. 
Making use of this lemma, we define the volume of a foliation cycle as follows. Let $\alpha$ be a foliation cycle and $\mu_{i}$ be as in Lemma 1. By Hahn's theorem, each $\mu_{i}$ has the unique decomposition as $\mu_{i}=\mu_{i+}-\mu_{i-}$ where $\mu_{i+}$ and $\mu_{i-}$ are nonnegative measures and each of them is singular with respect to the other. Let $\bar{\mu}_{i}=\mu_{i+}+\mu_{i-}$.

DEFINITION.

$$
\operatorname{volume}(\alpha)=\sum_{i} \int_{D_{i}^{m-p}}\left|\int_{P_{z}} \lambda_{i} v\right| d \bar{\mu}_{i}(z) .
$$

Here $v$ denotes the volume form on each leaf induced by the Riemannian metric.

It is easy to see that this definition is independent of the choice of distinguished charts and partitions of unity. Thus we have

$$
\operatorname{volume}\left(\left(f_{*}\right)^{n} \alpha\right)=\sum_{i} \int_{D_{i}^{m-p}}\left|\int_{P_{z}} \lambda_{i}\left(f^{*}\right)^{n} v\right| d \bar{\mu}_{i}(z) .
$$

The following lemma is immediate by this formula and that in Claim.

LEMMA 2. For any foliation cycle $\alpha$, we have

$$
\rho(f) \geq \underset{n}{\limsup } \frac{1}{n} \log \left(\operatorname{volume}\left(\left(f_{*}\right)^{n} \alpha\right)\right)
$$

2. Proof of theorems. Let $\hat{P}(x)=\bigcup_{x \in P_{z}} P_{z}$ and $\hat{B}_{r}(x)=B_{r}(x) \cap \hat{P}(x)$.

LEMMA 3. If one takes $\delta$ sufficiently small, then, for every $x \in M$,

$$
f\left(\hat{B}_{\delta}(x)\right) \cap B_{\delta}(f(x)) \subset \hat{B}_{\delta}(f(x)) \text {. }
$$

Proof. Since $\hat{P}(x)$ is a neighborhood of $x$ in the leaf $L$, there is a $\delta^{\prime}>0$ such that $f\left(D_{\delta^{\prime}}(x)\right) \subset \hat{P}(f(x))$. Take $\delta$ small enough that $\hat{B}_{\delta}(x) \subset D_{\delta^{\prime}}(x)$. Then one gets $f\left(\hat{B}_{\delta}(x)\right) \cap B_{\delta}(f(x)) \subset \hat{P}(f(x)) \cap B_{\delta}(f(x))=\hat{B}_{\delta}(f(x))$. Remark that, by the compactness, one can take $\delta^{\prime}$ and $\delta$ uniformly. Thus we have proved the required result.

Now we prove Theorem 1. If $\rho(f) \leq 0$, there is nothing to prove. Otherwise, it suffices to show that for every positive $\rho$ with $\rho<\rho(f), h(f) \geq \rho$. Fix such a $\rho$ and let $\delta$ be as in Lemma 3. Also fix $r$ small enough that for every $x \in M$, $D_{2 r}(x) \subset \hat{B}_{\delta}(x)$. Then, if $y \in D_{r}(x)$, we have that $D_{r}(x) \subset D_{2 r}(y) \subset \hat{B}_{\delta}(y)$.

LEMMA 4. For every positive integer $n_{0}$, there exists an integer $n \geq n_{0}$ and $x \in M$ satisfying

$$
\operatorname{volume}\left(f^{n}\left(D_{r}(x)\right)\right) \geq \exp (n \rho) \text {. }
$$

Proof. Immediate by the definition of $\rho(f)$.

Let $n$ and $D=D_{r}(x)$ be as in Lemma 4 and $s$ be $s_{f}\left(n+1, \frac{\delta}{2}\right)$. Take a subset $\left\{x_{1}, \ldots, x_{s}\right\}$ of $M$ which $\left(n+1, \frac{\delta}{2}\right)$-spans $M$ and put $X_{j}=\left\{y \in D ; d\left(f^{i}\left(x_{j}\right), f^{i}(y)\right)<\right.$ $\left.\frac{\delta}{2}, i=0, \ldots, n\right\}$. We may assume that $X_{1} \neq \emptyset, \ldots, X_{s^{\prime}} \neq \emptyset$ and $X_{s^{\prime}+1}=\cdots=$ $X_{s}=\emptyset$. Take $y_{j} \in X_{j}$ for $j=1, \ldots, s^{\prime}$. Then we have $f^{k}\left(X_{j}\right) \subset B_{\delta}\left(f^{k}\left(y_{j}\right)\right)$ for $k=0, \ldots, n$. Therefore, by the fact $X_{j} \subset D \subset \hat{B}_{\delta}\left(y_{j}\right)$, using Lemma 3 repeatedly, we get that $f^{n}\left(X_{j}\right) \subset \hat{B}_{\delta}\left(f^{n}\left(y_{j}\right)\right)$. Let $K$ be an upper bound for the volumes of 
plaques. Then, for every $x \in M$, volume $\left(\hat{B}_{\delta}(x)\right) \leq \operatorname{volume}(\hat{P}(x)) \leq N K$. So, by Lemma $4, s_{f}\left(n+1, \frac{\delta}{2}\right) \geq s^{\prime} \geq \frac{1}{N K} \exp (n \rho)$. Since we can take $n$ arbitrarily large,

$$
h(f) \geq \limsup _{n} \frac{1}{n+1} \log \left(s_{f}\left(n+1, \frac{\delta}{2}\right)\right) \geq \rho .
$$

This completes the proof of Theorem 1 .

COROLlaRY 1. For any foliation cycle $\alpha$, we have

$$
h(f) \geq \underset{n}{\lim \sup } \frac{1}{n} \log \left(\operatorname{volume}\left(\left(f_{*}\right)^{n} \alpha\right)\right) \text {. }
$$

ProOF. Immediate by Theorem 1 and Lemma 2.

COROLLARY 2. For any foliation cycle $\alpha$ and any $p$-form $\eta$, we have

$$
h(f) \geq \underset{n}{\lim \sup } \frac{1}{n} \log \left|\left\langle\left(f_{*}\right)^{n} \alpha, \eta\right\rangle\right| .
$$

ProOF. By the compactness of $M$, there is a constant $C$ such that for every $p$-dimensional Dirac current $\delta_{x}$ tangent to $\mathcal{F},\left|\left\langle\delta_{x}, v\right\rangle\right| \geq C\left|\left\langle\delta_{x}, \eta\right\rangle\right|$ where $v$ is the volume form. Then we have

$$
\begin{aligned}
\operatorname{volume}\left(\left(f_{*}\right)^{n} \alpha\right) & \geq \sum_{i} \int_{D_{i}^{m-p}}\left|\int_{P_{z}} \lambda_{i}\left(f^{*}\right)^{n}(C \eta)\right| d \bar{\mu}_{i}(z) \\
& \geq C \sum_{i}\left|\int_{D_{i}^{m-p}}\left(\int_{P_{z}} \lambda_{i}\left(f^{*}\right)^{n} \eta\right) d \mu_{i}(z)\right| \\
& \geq C\left|\left\langle\left(f_{*}\right)^{n} \alpha, \eta\right\rangle\right| .
\end{aligned}
$$

This and Corollary 1 imply the required result.

It is easy to see that Theorem 2 is an immediate corollary to the above result. Thus we have proved everything required.

Finally we remark that Theorem 1 holds also for continuous mappings from $M$ to itself which preserve $\mathcal{F}$. Here we say that $f$ preserves $\mathcal{F}$ if for any leaf $L$ of $\mathcal{F}$, there is a leaf $L^{\prime}$ such that $f(L) \subset L^{\prime}$.

\section{REFERENCES}

1. R. Bowen, Entropy for group endomorphisms and homogeneous spaces, Trans. Amer. Math. Soc. 153 (1971), 401-414.

2. J. Plante, Foliations with measure preserving holonomy, Ann. of Math. (2) 102 (1975), 327-361.

3. M. Shub, Dynamical systems, filtrations and entropy, Bull. Amer. Math. Soc. 80 (1974), $27-41$.

4. D. Sullivan, Cycles for the dynamical study of foliated manifolds and complex manifolds, Invent. Math. 36 (1976), 225-255.

Department of Mathematics, FaCUlty of SCIENCE, UNiversity of TOKyo, HONGO, TOKYO, JAPAN 